\section{TM6SF2 and fat metabolism}

Two recent studies identified TM6SF2 as a regulator of liver triglyceride content (Nat. Genet. 46, 352-356, 2014) and plasma total cholesterol levels (Nat. Genet. 46, 345-351, 2014) through exome-based association analyses and functional experiments in mice. In independent work, Ferdinand van't Hooft and colleagues (Proc. Natl. Acad. Sci. USA doi:10.1073/pnas.1323785111, 4 June 2014) performed a series of subcellular localization, knockdown and overexpression studies in cultured cells to gain further insights into TM6SF2 function. Using confocal microscopy, they observed localization of GFP-tagged TM6SF2 to the endoplasmic reticulum in two human hepatoma cell lines. They further showed that knockdown of TM6SF2 in both cell lines resulted in reduced secretion of triglyceride-rich lipoproteins and increased cellular triglyceride levels. At the subcellular level, they observed a marked increase in lipid droplet area, which could be attributed to increases in both the number and average size of lipid droplets. Conversely, they found that overexpression of TM6SF2 caused a decrease in the number and average size of lipid droplets. These results suggest a role for TM6SF2 in regulating the supply of lipids for the synthesis of triglyceride-rich lipoproteins.

\section{LDHA deregulation in glioma}

Tumor cells with neomorphic mutations in IDH1 and IDH2 gain the ability to produce 2 -hydroxyglutarate (2-HG), a metabolite involved in the degradation of HIF1a. These mutations are associated with better prognosis in glioma due to downregulation of the HIF1 a targets necessary for the switch to aerobic glycolysis. Now, J. Gregory Cairncross and colleagues report that $I D H 1$ and $I D H 2$ mutations in glioma lead to the downregulation of a key metabolic gene, $L D H A$ (encoding lactate dehydrogenase A), through DNA methylation of its promoter (Neuro. Oncol. 16, 686-695, 2014). Using glioma-derived brain tumor stem cells with mutation in $I D H 1$ or $I D H 2$, they found that cell lines that had lost the mutated IDH1 or IDH2 allele continued to hypermethylate the LDHA promoter and underexpress $L D H A$, even though 2-HG was no longer produced. They further analyzed glioma data from the Repository of Molecular Brain Neoplasia Data and found that LDHA is one of the most downregulated genes in $I D H 1$ - and $I D H 2$-mutant gliomas and that patients with low expression of $L D H A$ have a median survival of 50 months, in comparison to 16 months for patients without the mutations. They also analyzed data from The Cancer Genome Atlas and found that IDH1- and IDH2-mutant glioblastomas are associated with high LDHA promoter methylation and low LDHA expression.

\section{Cell of origin of hairy cell leukemia}

Hairy cell leukemia (HCL) is a B cell malignancy in which leukemic cells express mature $B$ cell surface markers and have clonal rearrangements of immunoglobulin genes. Although a somatic $\mathrm{BRAF}^{\mathrm{V} 600 \mathrm{E}}$ alteration occurs in nearly all cases of HCL, the cell of origin of this type of leukemia has not been definitively identified. Now, Omar Abdel-Wahab, Christopher Park and colleagues identify hematopoietic stem cells (HSCs) as the preleukemic cell of origin for HCL (Sci. Transl. Med. 6, 238ra71, 2014).

Written by Orli Bahcall, Brooke LaFlamme, Emily Niemitz \& Kyle Vogan
They used flow sorting of 14 HCL clinical samples and 3 age-matched controls to isolate cell populations along the hematopoietic hierarchy and assayed for the $\mathrm{BRAF}^{\mathrm{V} 600 \mathrm{E}}$ alteration in each sorted fraction, identifying $\mathrm{BRAF}^{\mathrm{V} 600 \mathrm{E}}$ in the HSC, pro-B cell and HCL cell populations from HCL samples. The presence of the alteration in phenotypically normal HSCs suggests that these cells are a preleukemic ancestral cell type. They also found that these BRAF ${ }^{\mathrm{V} 600 \mathrm{E}}$-bearing HSCs did not carry additional mutations present in leukemic cells. The authors then used mouse models to show that expression of $\mathrm{BRAF}^{\mathrm{V} 600 \mathrm{E}}$ in HSCs caused a lethal hematopoietic disorder similar to HCL, whereas targeting its expression to the $\mathrm{B}$ cell lineage caused no hematopoietic abnormalities.

EN

\section{Profiling osteogenesis}

Leonardo Meza-Zepeda and colleagues report the genomic and epigenomic profiles of a mesenchymal stem cell (MSC) line before and after differentiation to the osteogenic lineage (Stem Cells doi:10.1002/ stem.1759, 4 June 2014). Using RNA sequencing, they found 1,462 genes upregulated and 1,695 genes downregulated 28 days after differentiation was initiated. A master regulator of osteogenesis, RUNX2, was among the significantly upregulated genes in their data set. They subsequently performed genome-wide profiling of RUNX2 binding sites, identifying 8,871 candidate sites and 2,166 target genes in MSCs differentiated in vitro. Among the binding sites were 97 microRNA genes, 824 cancerrelated genes and 467 genes related to growth and proliferation. They further validated RUNX2 binding sites in primary NHOst osteoblasts. They also documented epigenetic changes through chromatin immunoprecipitation and sequencing (ChIP-seq) for five histone modifications, finding that trimethylation of lysine 4 on histone $\mathrm{H} 3$ (H3K4me3) was the most common differentially regulated histone modification. Finally, through filtering of candidate regions and in vitro silencing experiments, they identified TEAD2 and GTF2I as novel regulators of osteogenesis. These data provide an important resource for researchers studying osteogenic differentiation and bone cancer development.

$B L$

\section{Sheep genome}

Brian Dalrymple and colleagues from The International Sheep Genomics Sequencing Consortium report a high-quality reference genome for the sheep, Ovis aries (Science 344, 1168-1173, 2014). They sequenced the genomes of two Texel breed sheep: a 6-month-old ewe and an inbred ram. They used high-density radiation hybrid and linkage maps to aid in assembly, with a total assembled length of $\sim 2.61 \mathrm{~Gb}$. They constructed a phylogenetic tree on the basis of single-copy orthologous genes across livestock and humans and dated the separation of sheep from goats at 4.3 million years ago (confidence interval of 3.2-5.7 million years ago), overlapping with the expansion of $\mathrm{C} 4$ grasses. They also performed RNA sequencing analysis of 94 samples from 40 tissues. Protein clustering analysis across mammalian species identified 73 subfamilies specific to and 321 expanded in the ruminant lineage. They also characterized expression of genes within the mammalian epidermal development complex (EDC) region, involved in the development of the rumen, skin and wool. They identified keratin genes highly expressed in the rumen, including TCHHL2, encoding a putative keratin cross-linking protein. LCE7A was highly expressed in skin and was suggested to be involved in wool development, along with components of fatty acid metabolism and lipid enzymes. 